\title{
A straightforward strategy for constructing zirconium metallocavitands
}

Shunfu Du ${ }^{1,2}$, Xuying Yu ${ }^{1,2}$, Guoliang Liu' ${ }^{2}$,Mi Zhou ${ }^{2}$,EI-Sayed M. El-Sayed ${ }^{2,3,4}$, Zhanfeng $\mathrm{Ju}^{2}$, Kongzhao Su${ }^{2}$, Daqiang Yuan ${ }^{2 *}$

${ }^{1}$ College of Chemistry, Fuzhou University, Fuzhou 350108, China.

${ }^{2}$ Key Laboratory of Optoelectronic Materials Chemistry and Physics, Fujian Institute of Research on the Structure of Matter, Chinese Academy of Science, Fuzhou, 50002, China.

${ }^{3}$ University of the Chinese Academy of Sciences, Beijing, P. R. China.

${ }^{4}$ Chemical Refining Laboratory, Refining Department, Egyptian Petroleum Research Institute, Nasr City, Cairo, Egypt. 


\section{Single Crystal X-ray Diffraction Analyses}

Single-crystal X-ray data of three metallocavitands were collected on a SuperNova Dual $\mathrm{Cu}$ at zero, Atlas diffractometer. The crystals were kept at $100 \mathrm{~K}$ or $150 \mathrm{~K}$ during data collection. The data were collected with a $\omega$-scan technique and an arbitrary $\varphi$-angle. Data reduction was performed with the CrysAlisPro package, and an analytical absorption correction was performed. The structures were solved by direct methods and refined by full-matrix least-squares on $\mathrm{F}^{2}$ with anisotropic displacement using the SHELXTL-2018 software package, and the non-H atoms were treated anisotropically, whereas the aromatic and hydroxy-hydrogen atoms were placed in calculated, ideal positions and refined as riding on their respective carbon or oxygen atoms. In these structures, free solvent molecules were highly disordered, and attempts to locate and refine the solvent peaks were unsuccessful. The diffused electron densities resulting from these residual solvent molecules were removed from the data set using the SQUEEZE routine of PLATON and refined further using the data generated. The contents of the solvent region are not represented in the unit cell contents in the crystal data. The details for data collection and refinement are listed in Table S1. Crystallographic data for the structures reported in this paper have been deposited in the Cambridge Crystallographic Data Center with CCDC Number: 2027347-2027351 for ZrMCs These data can be obtained free of charge (http://www.ccdc.cam.ac.uk/data_request/cif). Full experimental details and crystallographic analysis are given in the Supplementary Information. 
Table S1. Crystal data and structure refinement for metallocavitands.

\begin{tabular}{|c|c|c|c|c|c|}
\hline & ZrMC-1 & $\mathrm{ZrMC-1- \textrm {NO } _ { 2 }}$ & ZrMC-1-NH & ZrMC-2 & ZrMC-3 \\
\hline \multirow[t]{2}{*}{ Formula } & $\mathrm{C}_{124} \mathrm{H}_{132} \mathrm{Cl}_{4} \mathrm{~N}_{4}$ & $\mathrm{C}_{108} \mathrm{H}_{90} \mathrm{Cl}_{4} \mathrm{~N}_{6} \mathrm{O}$ & $\mathrm{C}_{113} \mathrm{H}_{111} \mathrm{Cl}_{8} \mathrm{~N}_{7}$ & $\mathrm{C}_{156} \mathrm{H}_{134} \mathrm{ClNNa}$ & $\mathrm{C}_{72} \mathrm{H}_{62} \mathrm{Cl}_{3} \mathrm{O}_{30.50}$ \\
\hline & $\mathrm{O}_{47} \mathrm{Zr}_{12}$ & ${ }_{52} \mathrm{Zr}_{12}$ & $\mathrm{O}_{41} \mathrm{Zr}_{12}$ & $\mathrm{O}_{94} \mathrm{~S}_{8} \mathrm{Zr}_{18}$ & $\mathrm{~S}_{4.50} \mathrm{Zr}_{9}$ \\
\hline Fw & 3666.77 & 3540.29 & 3601.32 & 5483.51 & 2486.81 \\
\hline Crystal system & triclinic & monoclinic & tetragonal & triclinic & triclinic \\
\hline Space group & $P-1$ & $C 2 / c$ & $P 4 / n$ & $P-1$ & $P-1$ \\
\hline $\mathrm{a}, \AA$ & $21.0474(7)$ & $29.2273(7)$ & $43.1315(5)$ & $16.5151(3)$ & $18.3536(4)$ \\
\hline $\mathrm{b}, \AA$ & $25.9505(9)$ & $25.6887(17)$ & $43.1315(5)$ & $25.8768(5)$ & $19.9632(6)$ \\
\hline $\mathrm{c}, \AA$ & $26.0736(9)$ & 29.4711(9) & 23.9511(3) & $33.2471(6)$ & $20.1336(6)$ \\
\hline $\mathrm{a}, \mathrm{deg}$ & $112.233(3)$ & 90 & 90 & $80.303(2)$ & $109.235(3)$ \\
\hline$\beta, \operatorname{deg}$ & $104.130(3)$ & $91.785(3)$ & 90 & $78.7510(10)$ & $99.037(2)$ \\
\hline$\gamma, \operatorname{deg}$ & $106.942(3)$ & 90 & 90 & $82.099(2)$ & $114.086(3)$ \\
\hline $\mathrm{V}, \AA^{3}$ & $11556.3(8)$ & $22116.5(17)$ & $44556.8(12)$ & $13655.7(5)$ & $5983.9(3)$ \\
\hline Z & 2 & 4 & 8 & 2 & 2 \\
\hline $\mathrm{Dc}, \mathrm{g} / \mathrm{cm}^{3}$ & 1.054 & 1.063 & 1.074 & 1.334 & 1.380 \\
\hline$\mu, \mathrm{mm}^{-1}$ & 5.102 & 5.339 & 5.699 & 6.670 & 8.007 \\
\hline Reflns collcted & 84644 & 56413 & 95298 & 113379 & 43379 \\
\hline $2 \theta$ range, deg & 3.097 to 73.538 & 3.738 to 76.582 & 3.436 to 73.515 & 3.486 to 72.688 & 3.861 to 72.379 \\
\hline $\mathrm{F}(000)$ & 3656 & 6976 & 14256 & 5410 & 2442 \\
\hline GOF on $\mathrm{F}^{2}$ & 1.009 & 1.014 & 1.054 & 1.020 & 1.047 \\
\hline $\begin{array}{l}\mathrm{R} 1 / \mathrm{wR} 2 \\
(\mathrm{I}>2(\mathrm{I}))\end{array}$ & $\begin{array}{l}R 1=0.0779, \\
w R 2=0.2052\end{array}$ & $\begin{array}{l}R 1=0.0641, \\
w R 2=0.1790\end{array}$ & $\begin{array}{l}R 1=0.0868 \\
w R 2=0.2347\end{array}$ & $\begin{array}{l}R 1=0.0687 \\
w R 2=0.1767\end{array}$ & $\begin{array}{l}R 1=0.0644 \\
w R 2=0.1736\end{array}$ \\
\hline $\begin{array}{l}\text { R1/ wR2 } \\
\text { (all data) }\end{array}$ & $\begin{array}{l}R 1=0.1200 \\
w R 2=0.2555\end{array}$ & $\begin{array}{l}R 1=0.1279, \\
w R 2=0.2238\end{array}$ & $\begin{array}{l}R 1=0.1359 \\
w R 2=0.2629\end{array}$ & $\begin{array}{l}R 1=0.0961 \\
w R 2=0.2022\end{array}$ & $\begin{array}{l}R 1=0.0892, \\
w R 2=0.1942\end{array}$ \\
\hline
\end{tabular}


(a)

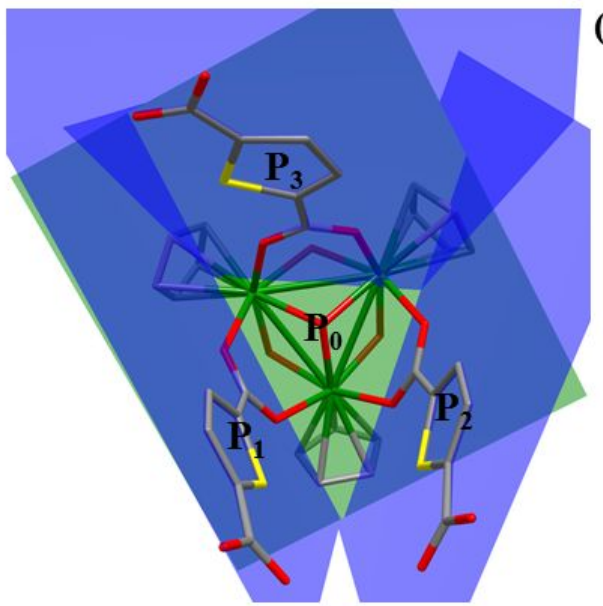

(b)

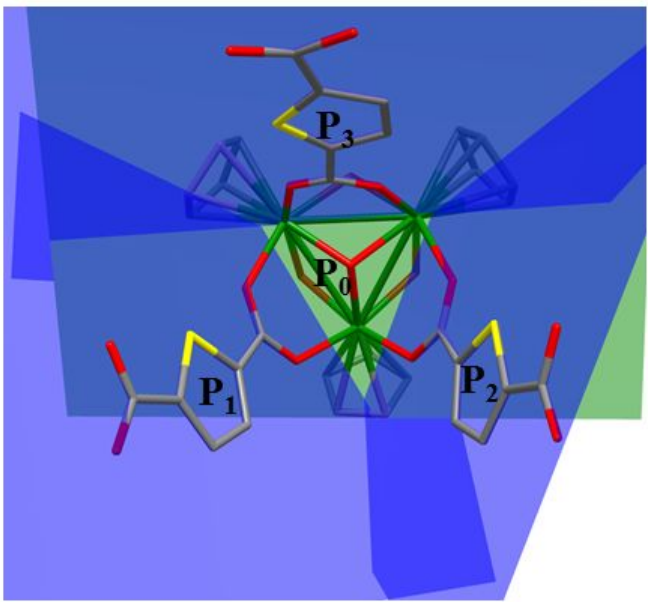

Figure S1 The green plane represents the plane on which the zirconium atoms are located, and the blue plane represents the plane on which the ligand is located. (a) the dihedral angles between $\mathrm{P}_{0}$ and $\mathrm{P}_{1}, \mathrm{P}_{0}$ and $\mathrm{P}_{2}, \mathrm{P}_{0}$ and $\mathrm{P}_{3}$ are $82.0^{\circ}, 79.0^{\circ}$, and 53.1 angles between $\mathrm{P}_{0}$ and $\mathrm{P}_{1}, \mathrm{P}_{0}$ and $\mathrm{P}_{2}, \mathrm{P}_{0}$ and $\mathrm{P}_{3}$ are $69.9^{\circ}, 67.3^{\circ}$, and $49.0^{\circ}$, respectively.

(a)

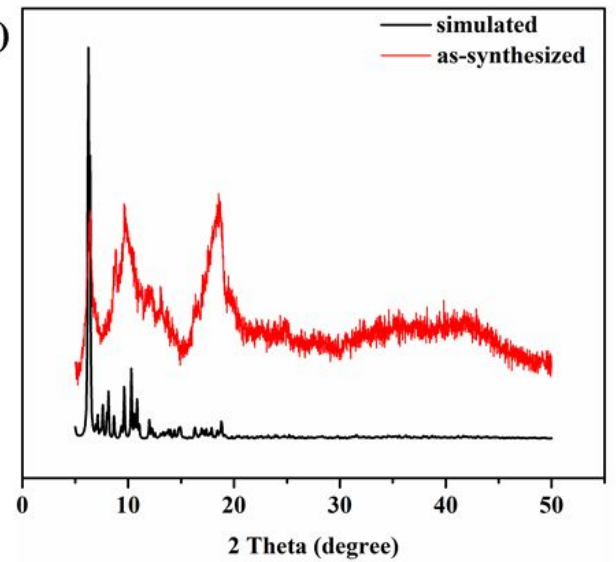

(b)

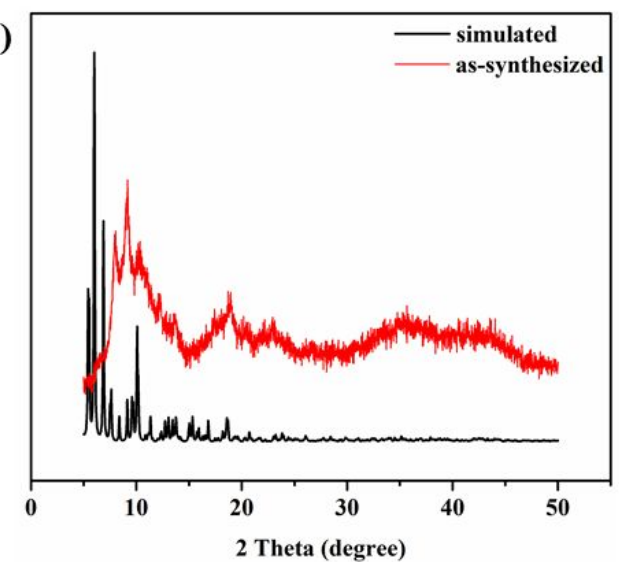

Figure S2 PXRD patterns of ZrMC-1 (a) and ZrMC-1-NO 2 (b).

(a)

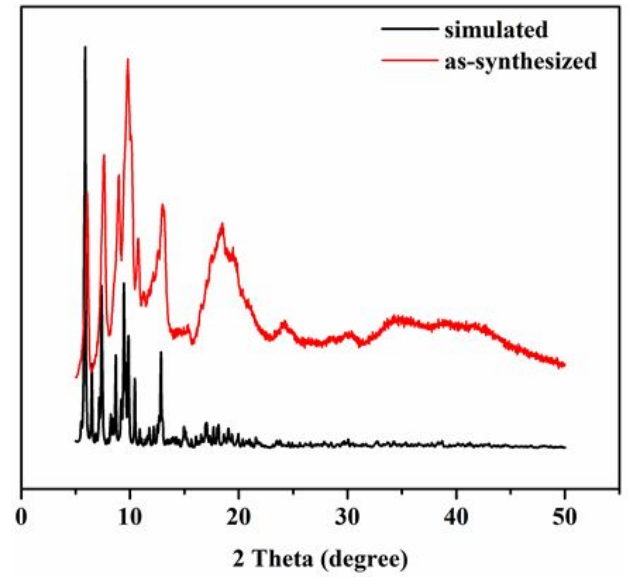

(b)

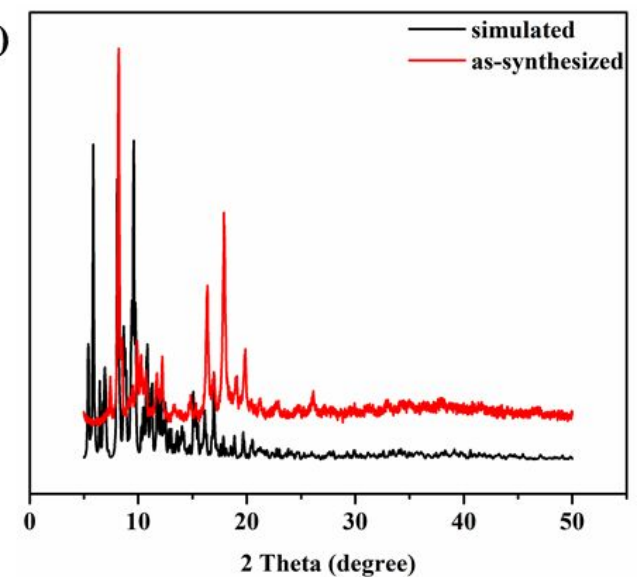

Figure S3 PXRD patterns of ZrMC-1-NH $\mathbf{N H}_{2}$ (a) and ZrMC-2 (b). 


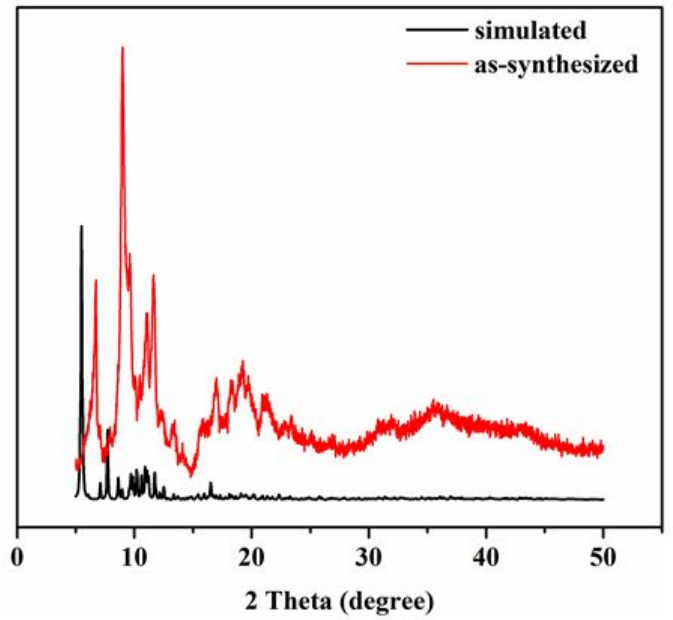

Figure S4 PXRD patterns of ZrMC-3.

(a)

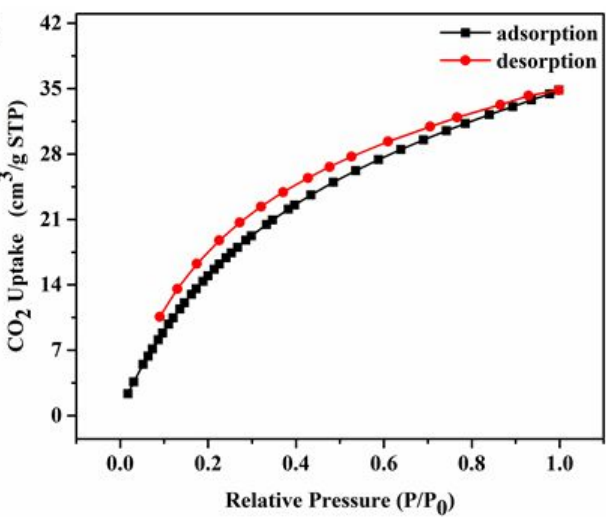

(b)

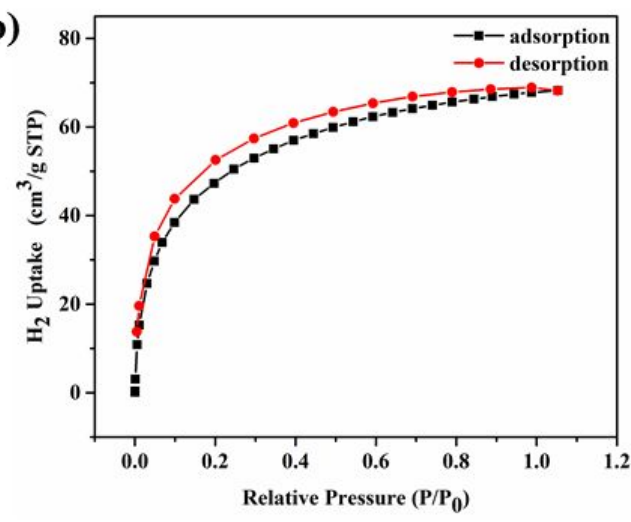

Figure S5 Experimental adsorption isotherms of ZrMC-2 for $\mathrm{CO}_{2}$ (a) and $\mathrm{H}_{2}$ (b) at 298 and $77 \mathrm{~K}$, respectively.

(a)

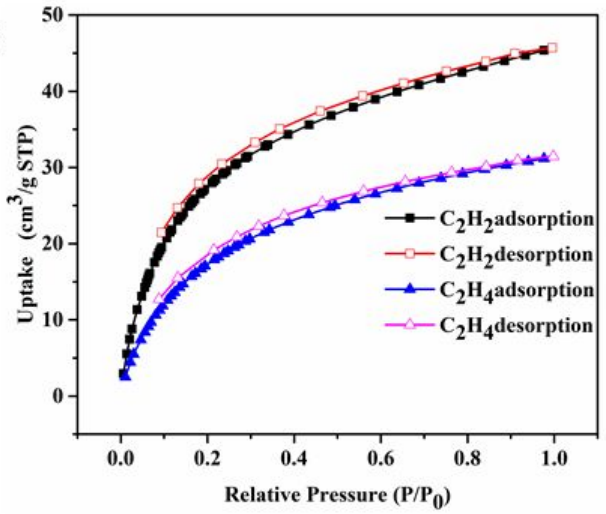

(b)

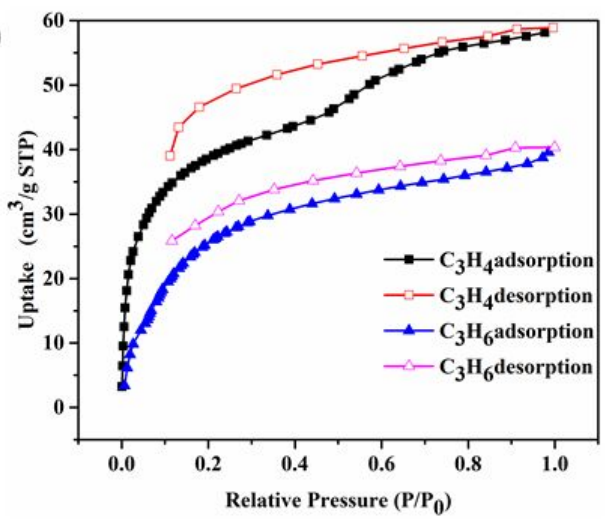

Figure S6 Experimental adsorption isotherms of ZrMC-2 for $\mathrm{C}_{2} \mathrm{H}_{2}$ and $\mathrm{C}_{2} \mathrm{H}_{4}(\mathrm{a})$ and $\mathrm{C}_{3} \mathrm{H}_{4}$ and $\mathrm{C}_{3} \mathrm{H}_{6}$ (b) at $298 \mathrm{~K}$. 